\title{
Perfil de natimortalidade de acordo com ordem de nascimento, peso e sexo de leitões
}

\author{
[Stillbirth pattern according to birth order, birth weight, and gender of piglets] \\ V.F. Borges ${ }^{1}$, M.L. Bernardi ${ }^{2}$, F.P. Bortolozzo ${ }^{1 *}$, I. Wentz $^{1}$ \\ ${ }^{1}$ Faculdade de Veterinária - UFRGS \\ Av. Bento Gonçalves, 9090 \\ 91540-000 - Porto Alegre, RS \\ ${ }^{2}$ Faculdade de Agronomia - UFRGS - Porto Alegre, RS
}

\begin{abstract}
RESUMO
Foram acompanhados 575 partos para avaliar a influência da ordem de nascimento, do sexo e do peso dos leitões na ocorrência de natimortos. Dos 7061 leitões, 90,2\%, 6,0\% e 3,8\% nasceram vivos, natimortos ou mumificados, respectivamente. O percentual de partos com natimortos foi 44,5\%. Partos com dois ou mais natimortos foram responsáveis por $63,1 \%$ das perdas por natimortalidade, embora tenham sido responsáveis por $17,2 \%$ das leitegadas. O percentual de natimortos aumentou com a ordem de nascimento; a maior taxa de natimortos, $21,7 \%$, ocorreu a partir da $14^{\mathrm{a}}$ ordem. A taxa de natimortalidade foi de $3,6 \%$ e $10,1 \%$ nos leitões de primeira a nona e de $10^{\mathrm{a}}$ a $13^{\mathrm{a}}$ ordem, respectivamente. Em leitões com até $500 \mathrm{~g}$ a taxa de natimortos foi de $52,1 \%$, mais alta que a de leitões mais pesados. Em leitões com 501 a $1200 \mathrm{~g}$ foi de $10,1 \%$, maior que entre os com mais de $1200 \mathrm{~g}(4,0 \%)$. Não houve efeito de sexo dos leitões na ocorrência de natimortos, que foi de 6,2\% e 5,8\% para machos e fêmeas, respectivamente. A natimortalidade é maior entre os leitões com baixo peso ou com ordem de nascimento elevada.
\end{abstract}

Palavras-chave: leitão, mortalidade perinatal, natimortos, peso ao nascimento

\begin{abstract}
To examine the influence of birth order, gender, and birth weight of piglets on stillbirth, records of 575 farrowings were analyzed. Out of 7,061 piglets, 90.2\%, 6.0\%, and 3.8\% were born alive, stillborns and mummified, respectively. The percentage of farrowings with stillborns was $44.5 \%$. Farrowings with two or more stillborns accounted for $63.1 \%$ of losses, although they produced $17.2 \%$ of the litters. Stillbirth rate increased with the increase of the birth order. Piglets born from the $14^{\text {th }}$ order onward had the highest stillbirth rate $(21.7 \%)$. Piglets born from the $1^{\text {st }}$ to the $9^{\text {th }}$ and from the $10^{\text {th }}$ to the $13^{\text {th }}$ order had stillbirth rates of $3.6 \%$ and $10.1 \%$, respectively. For piglets weighting up to 500g, the stillbirth rate was $52.1 \%$, which was higher than that recorded for heavier piglets. Piglets weighting from 501 to $1,200 \mathrm{~g}$ had a stillbirth rate of 10.1\%, which was higher than that observed for piglets weighing more than 1,200g (4.0\%). There was no effect of gender of piglets on stillbirth occurrence, which reachead $6.2 \%$ and $5.8 \%$ for males and females, respectively. The stillbirth occurrence was higher for low weight piglets or higher birth order.
\end{abstract}

Keywords: piglets, perinatal mortality, stillborn, birth weight

\section{INTRODUÇ̃̃O}

Na suinocultura, a produtividade é comumente medida pelo número de leitões desmamados por fêmea ao ano (Dial et al., 1992; Muirhead e Alexander, 1997). Nessa espécie, as perdas pré- natais representam 30 a $40 \%$ do número de ovulações e podem ser divididas em falhas de fecundação, mortalidade embrionária e mortalidade fetal (Meredith, 1995). A mortalidade embrionária e fetal pode variar de 20

Recebido em 12 de abril de 2007

Aceito em 27 de junho de 2008

*Autor para correspondência (corresponding author)

E-mail: fpbortol@ufrgs.br 
a $30 \%$ e de 5 a $15 \%$, respectivamente (Van Der Lende, 2000).

A importância da sobrevivência fetal em suínos tem aumentado devido ao incremento na taxa de ovulação e provável limitação da capacidade uterina. Analisando dados de várias publicações referentes à sobrevivência pré-natal, Van Der Lende e Schoenmaker (1990) observaram que a mesma não é alterada significativamente com o aumento do número de ovulações, até os 35 dias de gestação, mas diminui significativamente, após esse período. Rebanhos comerciais com altas taxas de ovulação podem ter altas taxas de mortalidade fetal. Partindo de um número inicial de 26,6 ovulações por estro, já no dia 25 de gestação, o número de conceptos era de 15,8 e, no dia 36 , baixava para 12,9 (Foxcroft et al., 2000).

A natimortalidade segue uma distribuição nãonormal, com a maioria dos partos sem a ocorrência de natimortos (Lucia Jr. et al., 2002). Em granjas brasileiras de suínos, têm sido relatados percentuais de $24,7 \%$ a $53,3 \%$ de partos com natimortos (Lucia Jr. et al., 2002; Schneider, 2002). A natimortalidade representa cerca de $25 \%$ das perdas que ocorrem entre o parto e o desmame (Cutler et al., 1992), estando entre as causas mais comuns de perdas no ciclo de produção de suínos (Cutler et al., 1992; Fraser et al., 1995). O índice aceitável de leitões natimortos está entre 3 e 5\% (Muirhead e Alexander, 1997), não devendo ultrapassar 7\% (Dial et al., 1992), embora taxas que variam de 3 a 10\% tenham sido relatadas (Cutler et al., 1992; Van Der Lende et al., 2001).

Aspectos como o intervalo entre nascimentos, a ordem de nascimento e o peso corporal dos leitões podem influenciar na ocorrência de natimortalidade. $\mathrm{O}$ intervalo entre nascimentos é maior entre um vivo e um natimorto, ou entre natimortos, do que entre vivos (Randall, 1972; Spicer et al., 1986). A anóxia fetal que ocorre durante o parto tem sido apontada como a maior causa de natimortalidade não infecciosa (Sprecher et al., 1974). Leitões com baixo peso são, em geral, oriundos de leitegadas de maior tamanho (Dial et al., 1992; Cutler et al., 1999; Holanda et al., 2005). $\mathrm{O}$ baixo peso os predispõem à natimortalidade, por terem menor vigor e maior risco de asfixia ao nascer (Spicer et al., 1986; Leenhouwers et al., 1999).

O objetivo deste trabalho foi caracterizar a ocorrência de natimortos de acordo com o sexo, a ordem de nascimento e o peso dos leitões, a partir de dados de partos acompanhados em granjas comerciais de suínos.

\section{MATERIAL E MÉTODOS}

O estudo foi realizado em quatro granjas comerciais de suínos, com plantéis de 2000 a 5000 matrizes em reprodução, no período de janeiro a maio de 2003. Foram acompanhados 575 partos de fêmeas de ordem de parto um a nove. Para cada parto acompanhado, foram registradas as ordens de parto da fêmea, número total de leitões nascidos, de nascidos vivos, de natimortos e de fetos mumificados. Foram registrados os dados referentes a somente um parto de cada fêmea. Os leitões foram pesados, individualmente, logo após o nascimento, sendo também registrados o sexo e a ordem de nascimento.

Os leitões natimortos foram necropsiados, e a natimortalidade foi classificada em pré-parto (PP), intraparto (IP) e pós-nascimento (PN). Os natimortos PP são aqueles mortos antes do início do parto, freqüentemente expulsos envolvidos nas membranas fetais, as quais sofrem descoloração e alterações degenerativas (Randall, 1972). Na necropsia, os órgãos internos estavam autolisados e pálidos, com evidências de embebição hemoglobínica (Sims e Glastonbury, 1996). Os natimortos IP são os que morrem durante o parto, apresentam aparência normal (Dial et al., 1992), podendo haver fragmentos de mecônio sobre a pele (Sims e Glastonbury, 1996). Na necropsia, havia congestão das vísceras e pulmões com consistência firme e coloração vermelho púrpura. Os pulmões de cada natimorto foram submersos em recipiente com água de modo a verificar se o leitão havia respirado ou não (Sims e Glastonbury, 1996). Os natimortos PP e IP foram aqueles cujos pulmões não flutuaram, considerados sem aeração pulmonar. Os natimortos PN apresentaram aeração pulmonar com conseqüente flutuação dos pulmões na água.

Ao nascimento, cada leitão foi registrado de acordo com a sua ordem de expulsão, do primeiro ao último. De acordo com a ordem de nascimento, independentemente do parto de origem, os leitões foram distribuídos em três classes. Na classe $1^{\circ}-9^{\circ}$ foram incluídos os leitões que nasceram da primeira até a nona ordem. $\mathrm{Na}$ classe $10^{\circ}-13^{\circ}$ foram incluídos os leitões que nasceram da décima até a décima terceira ordem e, na classe $\geq 14^{\circ}$, aqueles que nasceram a partir da décima quarta ordem. Também foram criadas quatro classes de peso ao nascimento, isto é, leitões com até $500 \mathrm{~g}$, com 501 a $1200 \mathrm{~g}, 1201$ a $2200 \mathrm{~g}$ e com mais de $2200 \mathrm{~g}$. 
A distribuição da freqüência de partos, de acordo com o número de natimortos e dos leitões, de acordo com o sexo, classes de ordem de nascimento e classes de peso ao nascimento, foram geradas pelo procedimento FREQ do SAS (User's..., 2000). Os percentuais de natimortos, de acordo com o sexo, classes de ordem de nascimento e classes de peso ao nascimento, foram comparados pelo teste Qui-quadrado ou teste exato de Fisher. Os fetos mumificados foram considerados no total de leitões nascidos para obter o percentual de nascidos vivos, natimortos e de mumificados. Nas demais análises, os 268 fetos mumificados não foram incluídos.

\section{RESULTADOS E DISCUSSÃO}

Dos 7061 leitões nascidos vivos ou natimortos, 6793 foram identificados conforme a ordem de nascimento, 6710 foram pesados e 6702 tiveram o sexo identificado. O tamanho da leitegada das fêmeas avaliadas foi de $12,2 \pm 3,5$ leitões, incluindo os vivos, natimortos e fetos mumificados. Do total de 7061 leitões, 6368 $(90,2 \%)$ nasceram vivos, $425 \quad(6,0 \%)$ eram natimortos e $268 \quad(3,8 \%)$ eram fetos mumificados. A taxa de natimortos observada situa-se entre as faixas de 4 a 10\% (Cutler et al., 1992) ou de 3 a 8\% (Van Der Lende et al., 2001) relatadas anteriormente. Dos 425 natimortos, 14 não puderam ser classificados de acordo com o momento de sua morte, sendo observados $13,4 \%$ (55/411) natimortos do tipo PP, 81,0\% (333/411) do tipo IP e 5,6\% (23/411) do tipo PN. Os valores percentuais de natimortos PP e IP estão próximos aos cerca de $10 \%$ e $75 \%$ relatados para leitões com morte antes do parto e durante o parto, respectivamente (Van Der Lende et al., 2001). Em seis granjas brasileiras, as variações nos valores percentuais de natimortos dos tipos PP, IP e PN observados em 908 partos (Schneider, 2002) foram de 9,1 a 39,1\%, de 51,2 a $77,3 \%$ e de 8,1 a $19,4 \%$, respectivamente.

Cerca de $60 \%$ dos partos ocorrem sem natimortos (Cutler et al., 1992). No presente estudo, dos 575 partos acompanhados, 44,5\% (256/575) tiveram natimortos, percentual que está acima dos $38,6 \%$ e $24,7 \%$ observados em granjas brasileiras, nas quais 101 e 373 partos foram respectivamente acompanhados (Lucia Jr. et al., 2002), mas abaixo de 53,3\% constatados em 908 partos (Schneider, 2002). Partos com dois ou mais natimortos foram responsáveis por $63,1 \%$ dos natimortos, mas representavam 17,2\% (99/575) dos partos (Tab. 1), confirmando o relato de que poucos partos são responsáveis pela maioria dos natimortos (Cutler et al., 1992). Partos com um natimorto diferiram $(\mathrm{P}<0,05)$ dos partos sem natimortos apenas em tamanho da leitegada (12,3 versus 11,5 leitões), mas não diferiram $(\mathrm{P}>0,05)$ em termos de ordem de parto, peso da leitegada e peso médio dos leitões. Por outro lado, a ordem de parto, o número de leitões e o peso da leitegada foram maiores, e o peso médio dos leitões foi menor $(\mathrm{P}<0,05)$ nos partos com dois ou mais natimortos, em comparação aos partos sem natimortos $(4,8$ versus 3,3 partos; 14,4 versus 11,5 leitões; 17,8 versus $15,6 \mathrm{~kg}$ e 1307 versus 1441g). O aumento da natimortalidade com o aumento da ordem de parto ou idade da mãe já foi relatado em outros estudos (Leenhouwers et al., 1999; Lucia Jr. et al., 2002; Santoro et al., 2003). O aumento da natimortalidade, em fêmeas mais velhas, pode estar associado a fatores como obesidade (Muirhead e Alexander, 1997), partos prolongados (Cutler et al., 1992) ou maior tamanho da leitegada (Dial et al., 1992).

Tabela 1. Distribuição de freqüência dos partos e dos natimortos de acordo com o número de natimortos por parto

\begin{tabular}{|c|c|c|c|c|}
\hline \multirow{2}{*}{$\begin{array}{l}\text { Número de } \\
\text { natimortos/ parto }\end{array}$} & \multicolumn{2}{|c|}{$\begin{array}{l}\text { Partos/total de partos } \\
\qquad(\mathrm{n}=575)\end{array}$} & \multicolumn{2}{|c|}{$\begin{array}{l}\text { Natimortos/total natimortos } \\
\qquad(\mathrm{n}=425)\end{array}$} \\
\hline & $\mathrm{n}$ & $\%$ & $\mathrm{n}$ & $\%$ \\
\hline 1 & 157 & 27,3 & 157 & 36,9 \\
\hline 2 & 59 & 10,3 & 118 & 27,8 \\
\hline 3 & 25 & 4,3 & 75 & 17,6 \\
\hline 4 & 9 & 1,6 & 36 & 8,5 \\
\hline 5 & 4 & 0,7 & 20 & 4,7 \\
\hline 6 & 1 & 0,17 & 6 & 1,4 \\
\hline 13 & 1 & 0,17 & 13 & 3,1 \\
\hline
\end{tabular}


$\mathrm{Na}$ Tab. 2, é apresentada a distribuição dos partos de acordo com o fato de terem iniciado ou terminado com o nascimento de natimortos. Dos 157 partos com um único natimorto, em apenas $5,1 \%$ (8/157) dos casos esse natimorto foi o primeiro leitão, contrastando com $36,9 \%$ (58/157) dos casos em que o natimorto nasceu por último. Foi também investigado qual era o percentual de natimortos que havia nascido após um outro natimorto. Dos 425 natimortos, 337 $(79,3 \%)$ nasceram após um leitão vivo enquanto $88(20,7 \%)$ sucederam o nascimento de um outro leitão natimorto. Ocorreram 48, 10, quatro e uma seqüências de dois, três, quatro e nove natimortos, respectivamente, envolvendo 35,5\% dos natimortos (151/425) e 22,3\% dos partos com natimortos (57/256). Em seis casos, havia mais de uma seqüência de natimortos, no mesmo parto (três partos com duas seqüências de dois natimortos; em dois partos uma seqüência de dois e outra de três natimortos; um parto com uma seqüência de nove e uma de quatro natimortos). Em partos com dois ou mais natimortos, em mais da metade deles houve seqüências de natimortos $(57,6 \% ; 57 / 99)$, as quais foram mais freqüentes nos partos com três ou mais natimortos, pois $85 \%$ deles $(34 / 40)$ tiveram pelo menos uma seqüência de dois natimortos. Esses 34 partos representavam 5,9\% do total de partos, mas foram responsáveis por $30,8 \%$ dos natimortos (131/425). Houve, também, casos em que seqüências de dois ou mais natimortos foram intercaladas por apenas um leitão vivo $(n=14)$ ou o nascimento de dois natimortos foi intercalado pelo nascimento de um leitão vivo $(n=12)$. Além disso, das 63 seqüências de nascimento de natimortos observadas, 71,4\% delas ocorreram a partir do nascimento do $10^{\circ}$ leitão. $\mathrm{O}$ conjunto dessas observações confirma relatos anteriores de que leitões nascidos no início do parto estão em sua maioria vivos, mas, à medida que o parto progride, a proporção de natimortos aumenta (Randall, 1972) ou, após 80\% dos leitões terem nascido, o número de natimortos aumenta (Cutler et al., 1992).

Tabela 2. Distribuição de freqüência dos partos de acordo com a ordem de nascimento dos leitões natimortos

\begin{tabular}{lccc}
\hline \multicolumn{1}{c}{ Tipo de parto } & $\begin{array}{c}\text { Número de } \\
\text { partos }\end{array}$ & $\begin{array}{c}\text { \% sobre o total de } \\
\text { partos }(\mathrm{n}=575)\end{array}$ & $\begin{array}{c}\text { \% sobre partos com } \\
\text { natimortos }(\mathrm{n}=256)\end{array}$ \\
\hline Com natimorto único e primeiro & 8 & 1,4 & 3,1 \\
Com natimorto único e último & 58 & 10,1 & 22,7 \\
Com natimorto único não sendo nem o & 91 & 15,8 & 35,5 \\
primeiro nem o último & & & 7,0 \\
Começando com natimorto & 18 & 3,1 & 43,4 \\
$\begin{array}{l}\text { Encerrando com natimorto } \\
\text { Com mais de um natimorto e terminando }\end{array}$ & 111 & 19,3 & 21,1 \\
com natimorto & 54 & 9,4 & \\
\hline
\end{tabular}

Ao efetuar a separação em classes por ordem de nascimento (Tab. 3), leitões da classe $\geq 14^{\circ}$ tiveram maior percentual $(\mathrm{P}<0,05)$ de natimortos que aqueles da classe $10^{\circ}-13^{\circ}$ que, por sua vez, tiveram maior percentual $(\mathrm{P}<0,05)$ que os leitões da classe $1^{\circ}-9^{\circ}$. Chama a atenção o fato de que houve o nascimento de 14 ou mais leitões em $34,4 \%$ dos partos (198/575), os quais estariam correndo maiores riscos de natimortalidade. À medida que aumentou a ordem de nascimento, o maior aumento no percentual de natimortos ocorreu no tipo intraparto. Estes resultados confirmam o fato de que a ocorrência de asfixia é maior com o aumento do tamanho da leitegada (Herpin et al., 2001). Sinais típicos de privação de oxigênio, freqüentemente associados com dano do cordão umbilical (Randall, 1972), estão provavelmente relacionados ao estiramento do cordão durante a expulsão do leitão, especialmente para aqueles localizados próximo à extremidade ovariana do útero (Fraser et al., 1995).

O destino dos fetos com crescimento retardado é a morte nos estágios finais da gestação ou o subdesenvolvimento após o nascimento (Wise et al., 1997). Aproximadamente 1/3 das leitegadas contém pelo menos um leitão que é substancialmente mais leve que os demais (Ashworth et al., 2001). Leitões natimortos têm menor peso que os vivos, comprovando o maior risco de natimortalidade em caso de baixo peso (Leenhouwers et al., 1999). No presente estudo, foi confirmado o maior peso $(\mathrm{P}<0,05)$ dos leitões 
nascidos vivos $(1395,3 \pm 343,8 \mathrm{~g} ; \mathrm{n}=6298)$ em comparação aos natimortos $(1181,0 \pm 424,6 \mathrm{~g}$; $\mathrm{n}=412$ ). O maior percentual de natimortos foi observado na classe de leitões que pesaram até $500 \mathrm{~g}$, sendo superior às classes de leitões mais pesados (Tab. 4). Estes dados confirmam relato anterior (Spicer et al., 1986) de que 25\% dos leitões com menos de $800 \mathrm{~g}$ são natimortos. Os leitões da classe 501-1200g tiveram maior percentual de natimortos que aqueles pesando acima de 1200g. Spicer et al. (1986) também constataram baixas taxas de natimortos $(1,7 \%$ e $2,5 \%$ ) entre os leitões com 1,4 a $1,5 \mathrm{~kg}$ e 1,8 a $1,9 \mathrm{~kg}$, respectivamente.

Tabela 3. Ocorrência de natimortos pré-parto (PP), intraparto (IP) e pós-nascimento (PN) de acordo com a ordem de nascimento dos leitões

\begin{tabular}{cccccc}
\hline $\begin{array}{c}\text { Ordem de } \\
\text { nascimento }\end{array}$ & $\begin{array}{c}\text { PP } \\
\mathrm{n}(\%)\end{array}$ & $\begin{array}{c}\text { IP } \\
\mathrm{n}(\%)\end{array}$ & $\begin{array}{c}\text { PN } \\
\mathrm{n}(\%)\end{array}$ & $\begin{array}{c}\text { Não identificados } \\
\mathrm{n}(\%)\end{array}$ & $\begin{array}{c}\text { Total de natimortos } \\
\mathrm{n}(\%)\end{array}$ \\
\hline $1^{\mathrm{o}}-9^{\mathrm{o}}$ & $30(0,6)$ & $130(2,7)$ & $10(0,2)$ & $6(0,12)$ & $176 / 4882(3,6) \mathrm{a}$ \\
$10^{\circ}-13^{\circ}$ & $20(1,4)$ & $109(7,7)$ & $9(0,6)$ & $5(0,35)$ & $143 / 1422(10,1) \mathrm{b}$ \\
$\geq 14^{\circ}$ & $5(1,0)$ & $94(19,3)$ & $4(0,8)$ & $3(0,61)$ & $106 / 489(21,7) \mathrm{c}$
\end{tabular}

Valores seguidos de letras diferentes são estatisticamente diferentes pelo teste $\chi^{2}(\mathrm{P}<0,05)$.

Tabela 4. Ocorrência de natimortos de acordo com as classes de peso e de ordem de nascimento dos leitões

\begin{tabular}{lcccc}
\hline \multirow{2}{*}{ Classes de peso } & $\begin{array}{c}\text { Natimortos } \\
\text { n/total }(\%)\end{array}$ & \multicolumn{3}{c}{ Classes de ordem de nascimento } \\
\cline { 3 - 5 } & $25 / 48(52,1) \mathrm{a}$ & $10 / 29(34,5) \mathrm{aA}$ & $6 / 10(60,0) \mathrm{aAB}$ & $\begin{array}{c}10^{\circ}-13^{\circ} \\
\mathrm{n} / \text { total }(\%)\end{array}$ \\
\hline Até 500g & n/total $(\%)$ \\
501-1200g & $198 / 1954(10,1) \mathrm{b}$ & $85 / 1387(6,1) \mathrm{bA}$ & $68 / 419(16,2) \mathrm{bB}$ & $45 / 148(30,4) \mathrm{bC}$ \\
Mais de 1200g & $189 / 4708(4,0) \mathrm{c}$ & $81 / 3433(2,4) \mathrm{cA}$ & $59 / 960(6,1) \mathrm{cB}$ & $49 / 315(15,6) \mathrm{cC}$
\end{tabular}

Valores seguidos de letras minúsculas distintas, na coluna, diferem pelo teste $\chi^{2}(\mathrm{P}<0,05)$.

Valores seguidos de letras maiúsculas distintas, na linha, dentro das classes de ordem de nascimento, diferem pelo teste de Fischer ou $\chi^{2}(\mathrm{P}<0,05)$.

Fetos mais leves e com possível comprometimento do peso ao nascer já podem ser identificados aos 30 dias de gestação (Wise et al., 1997). Além disto, leitões com menor peso ao nascer já eram fetos com menor tamanho, aos 40 dias de gestação (Chen e Dziuk, 1993), mostrando que o espaço uterino limitado não parece ser o principal determinante de um crescimento fetal inadequado (Ashworth et al., 2001). Aos 104 dias de gestação, fetos suínos mais leves tiveram menores concentrações de IGF-I e colesterol, refletindo diminuição do potencial de crescimento, nutrição subótima e inadequado desenvolvimento do sistema nervoso central (Wise et al., 1997). Fetos de menor tamanho teriam desenvolvimento assimétrico dos órgãos, placentas menores, baixa concentração plasmática de aminoácidos, alta concentração plasmática de cortisol, capacidade placentária reduzida para o transporte de leucina e atraso na troca dos transportadores placentários de aminoácidos (Ashworth et al., 2001). O fato de leitões com baixo peso apresentarem comprometimento da fisiologia muscular, cardíaca e renal (Bauer et al., 2003) sugere que as alterações que comprometem a função desses órgãos ocorrem já na vida intrauterina, o que explica a menor probabilidade de esses leitões serem capazes de superar as adversidades inerentes ao nascimento em si, aumentando suas chances de serem natimortos. Considerando que um retardo no crescimento intrauterino dificilmente será totalmente compensado por uma melhor assistência ao parto, as tentativas de aliviar um desenvolvimento fetal inadequado devem visar à promoção da uniformidade dentro da leitegada, durante toda a prenhez (Ashworth et al., 2001).

É conhecido o fato de que leitegadas grandes estão associadas com baixo peso ao nascer (Holanda et al., 2005), aumento nas taxas de 
natimortos e de mumificados, além da diminuição da sobrevivência pré-desmame (Dial et al., 1992; Cutler et al., 1999; Leenhouwers et al., 1999). Ficou claro, no presente estudo, que o baixo peso do leitão e a ordem de nascimento $>10$ estiveram associados a maiores taxas de natimortalidade. $\mathrm{O}$ efeito desses dois fatores pode ser melhor avaliado nos dados apresentados na Tab. 4, pois, dentro de cada classe de ordem de nascimento, o percentual de natimortos diminui à medida que aumenta o peso dos leitões e, dentro de cada classe de peso, aumenta o percentual de natimortos à medida que aumenta a ordem de nascimento. Além disso, o impacto do menor peso ao nascer foi evidente nas leitegadas maiores, visto que os 48 leitões que pesaram até $500 \mathrm{~g}$, dos quais $52,1 \%$ foram natimortos, todos pertenciam a leitegadas com, no mínimo, 10 leitões.

De 6702 leitões com sexo identificado, 3493 $(52,1 \%)$ eram do sexo masculino e $3209(47,9 \%)$ do sexo feminino. Não houve diferença $(\mathrm{P}=0,48)$ no percentual de natimortos entre os leitões do sexo masculino $(6,2 \% ; 218 / 3493)$ e feminino $(5,8 \% ; 187 / 3209)$. Não houve diferença $(\mathrm{P}>0,05)$ na distribuição de fêmeas e machos, de acordo com a ordem de nascimento, o que confirma o caráter aleatório desse evento, fazendo com que a ordem de nascimento não favorecesse um dos dois sexos.

\section{CONCLUSÕES}

$\mathrm{O}$ peso ao nascimento abaixo de $1200 \mathrm{~g}$ e a ordem de nascimento $>10$ aumentam a natimortalidade. A natimortalidade não é afetada pelo sexo do leitão.

\section{REFERÊNCIAS BIBLIOGRÁFICAS}

ASHWORTH, C.J.; FINCH, A.M.; PAGE, K.R. et al. Causes and consequences of fetal growth retardation in pigs. Reproduction, v.58, suppl., p.233-246. 2001.

BAUER, R.; WALTER, B.; BRUST, P. et al. Impact of asymmetric intrauterine growth restriction on organ function in newborn piglets. Eur. J. Obstet. Gynecol. Reprod. Biol., v.110, p.40-49, 2003.
CHEN, Z.Y.; DZIUK, P.J. Influence of initial length of uterus per embryo and gestation stage on prenatal survival, development, and sex ratio in the pig. J. Anim. Sci., v.71, p.1895-1901, 1993.

CUTLER, R.S.; FAHY, V.A.; SPICER, E.M. Preweaning mortality. In: LEMAN, A.D.; STRAW, B.E.; MENGELING, W.L. et al. (Eds). Diseases of swine. London: Wolfe, 1992. p.847860.

CUTLER, R.S.; FAHY, V.A.; SPICER, E.M. et al. Preweaning mortality. In: STRAW, B.E.; D'ALLAIRE, S.; MENGELING, W.L. et al. (Eds). Diseases of swine. Ames: Iowa State University, 1999. p.985-1002.

DIAL, G.D.; MARSH, W.E.; POLSON, D.D. et al. Reproductive failure: differential diagnosis. In: LEMAN, A.D.; STRAW, B.E., MENGELING, W.L. et al (Eds). Diseases of swine. London: Wolfe, 1992. p.88-137.

FOXCROFT, G.; WILSON, M.; VONNAHME, $\mathrm{K}$. et al. Changing patterns of prenatal loss in commercial sow genotypes. In: INTERNATIONAL CONGRESS ON ANIMAL REPRODUCTION, 14., 2000, Stockholm. Proceedings... Stockholm, 2000. p.28.

FRASER, D.; PHILLIPS, P.A.; THOMPSON, B.K. et al. Behavioural aspects of piglet survival and growth. In: VARLEY, M.A. (Ed). The neonatal pig. Development and survival. Guilford: Cab International, 1995. p.287-312.

HERPIN, P.; HULIN, J.C.; LE DIVIDICH, J. et al. Effect of oxygen inhalation at birth on the reduction of early postnatal mortality in pigs. $J$. Anim. Sci., v.79, p.5-10, 2001.

HOLANDA, M.C.R.; BARBOSA, S.B.P.; SAMPAIO, I.B.M. et al. Tamanho da leitegada e pesos médios, ao nascer e aos 21 dias de idade, de leitões da raça Large White. Arq. Bras. Med. Vet. Zootec., v.57, p.539-544, 2005.

LEENHOUWERS, J.I.; VAN DER LENDE, T.; KNOL, E.F. et al. Analysis of stillbirth in different lines of pig. Livest. Prod. Sci., v.57, p.243-253, 1999.

LUCIA JR., T.; CORREA, M.N.; DESCHAMPS, J.C. et al. Risk factors for stillbirth in two swine farms in the south of Brazil. Prev. Vet. Med., v.53, p.285-292, 2002. 
MEREDITH, M.J. Pig breeding and infertility. In: MEREDITH, M.J. (Ed). Animal breeding and infertility. Cambridge: Blackwell Science, 1995. p.278-353.

MUIRHEAD, M.R.; ALEXANDER, T.J.L. (Eds). Managing pig health and the treatment of disease. A reference for the farm. Sheffield: $5 \mathrm{M}$ Enterprises, 1997. p.133-226.

RANDALL, G.C.B. Observations on parturition in the sow. II. Factors influencing stillbirth and perinatal mortality. Vet. Rec., v.90, p.183-186, 1972.

SANTORO, K.R.; BARBOSA, S.B.P.; HOLANDA, M.C.R. Modelos de predição da natimortalidade em suínos. R. Bras. Zootec., v.32, p.1131-1140, 2003.

SCHNEIDER, L.G. Natimortalidade suina em granjas industriais: distribuição, qualidade dos registros do parto e causas associadas à natimortalidade pré-parto, intraparto e pósnascimento. 2002. 96f. Dissertação (Mestrado) Faculdade de Veterinária, Universidade Federal do Rio Grande do Sul, Porto Alegre.

SIMS, L.D.; GLASTONBURY, J.R.W. Neonatal mortality. In: SIMS, L.D.; GLASTONBURY, J.R.W. (Eds). Pathology of the pig. Barton: The pig Research and Development Corporation, 1996. p.423-432.

SPICER, E.M.; DRIESEN, S.J.; FAHY, V.A. et al. Causes of preweaning mortality on a large intensive piggery. Aust. Vet. J., v.63, p.71-75, 1986.

SPRECHER, D.J.; LEMAN, A.D.; DZIUK, P.D. et al. Causes and control of swine stillbirths. $J$. Am. Vet. Med. Assoc., v.165, p.698-701, 1974.
USER'S guide: statistical analysis system. Release 8.0. Cary, NC: SAS Institute, 2000.

VAN DER LENDE, T. Embryonic and fetal mortality in swine: causes, consequences and how to prevent these losses. In: SIMPÓSIO INTERNACIONAL DE REPRODUÇÃO E INSEMINAÇÃO ARTIFICIAL EM SUÍNOS, 7., 2000, Foz do Iquaçu. Anais... Foz do Iguaçu, 2000. p.243-252.

VAN DER LENDE, T.; KNOL, E.F.; LEENHOUWERS, J.I. et al. Prenatal development as a predisposing factor for perinatal losses in pigs. Reproduction, v.58, suppl., p.247-261. 2001.

VAN DER LENDE, T.; SCHOENMAKER, G.J.W. The relationship between ovulation rate and litter size before and after day 35 of pregnancy in gilts and sows: an analysis of published data. Livest. Prod. Sci., v.26, p.217229, 1990.

WISE, T.; CHRISTENSON, R.K. Relationship of fetal position within the uterus to fetal weight, placental weight, testosterone, estrogens, and thymosin $\beta 4$ concentrations at 70 and 104 days of gestation in swine. J. Anim. Sci., v.70, p.27872793, 1992.

WISE, T.; ROBERTS, A.J.; CHRISTENSON, R.K. et al. Relationships of light and heavy fetuses to uterine position, placental weight, gestational age, and fetal cholesterol concentrations. J. Anim. Sci., v.75, p.2197-2207, 1997. 